\title{
A Study on Numerical Simulation for Functional Microbial Flooding
}

\author{
Jianlong Xiu ${ }^{1,2, a}$, Weiyao Zhu ${ }^{1, b}$, Ying Guo ${ }^{2, c}$, Li Yu ${ }^{2, d}$ \\ ${ }^{1}$ Civil and Environmental Engineering School ,University of Science and Technology Beijing, \\ Beijing, 100083, China \\ ${ }^{2}$ Research Institute of Petroleum Exploration \& Development -Langfang, Petrochina, Langfang, \\ 065007,China \\ aemail: yisheng_218@163.com, bemail: \\ weiyaook@sina.com, cemail:gu082y@163.com, demail:yuli69@petrochina.com.cn
}

\begin{abstract}
Keywords: Functional microorganism; Indigenous microorganism; Microbial enhanced oil recovery; Compatibility; Mathematical model; Numerical simulation
\end{abstract}

\begin{abstract}
By studying on the transport laws and reaction dynamics for microorganisms in porous media, a comprehensive three-dimensional, three -phase mathematical model was established to reflect the mechanisms for microbial enhanced oil recovery. The components in this model included oil, gas, water, microorganism 1(exogenous microbe), microorganism 2 (indigenous microbe), nutrient, metabolite 1(surfactant), metabolite 2(polymer) and metabolite 3(gas). The numerical model has elaborated the convection, dispersion, diffusion, chemotaxis, adsorption and compatibility for microorganisms in porous media. On the basis of Monod model growth kinetic equation, interaction factors of two microbes were introduced for the first time to reflect mutual effect of indigenous microbes with endogenous microorganisms in oil reservoir. Meanwhile, equations about production for products and consumption for nutrient were modified to represent the metabolism and enhanced oil recovery for injected functional microbes accurately in the reservoir environment. The results make a great contribution to learn metabolic regulation of injected functional microbes in oil reservoir.
\end{abstract}

\section{Introduction}

By injecting functional microorganism and nutrients into the reservoir, with the local enrichment of microorganism, positioned reproduce and metabolism, some materials were produced such as bio-surfactant, bio-polymer, gas, acid, which were conducive to the oil displacement. These materials could change the concreted reservoir rock physical and chemical properties of crude oil, so as to improve oil recovery [1]. Microbial enhanced oil recovery (MEOR) has two different types called exogenous and endogenous methods based on different microbial sources [2].

For indigenous MEOR, efficient functional bacteria has played an important role in the process of microbial oil displacement. On the one hand, effective functional bacteria can make up the deficiency of low concentration endogenous bacteria in reservoir, on the other hand, it can activate the endogenous fungus [3]. The study on reservoir metabolic characteristics for efficient functional bacteria is quite conducive to microbial oil displacement system and spot design process scheme. Therefore, microbial reaction kinetics model was revised in this paper from the compatibility of injected exogenous bacteria and endogenous bacteria in reservoir. The quantitative characterization of microbial oil displacement was implemented in injection process of functional bacteria. By comparing the microorganism distribution and ultimate oil displacement efficiency from the new model with the previous model, this model can actually reflect growth and breeding for microorganisms under the condition of reservoir, as well as the oil displacement characteristics.

\section{Mathematical Model Theory}

Injected functional bacteria and endogenous bacteria may be in a partial, symbiotic, mutually beneficial symbiosis or quorum sensing relationship. Current research mainly focused on nitrate 
reducing bacteria competitive inhibition with sulfate reducing bacteria in oil reservoir. These common functional bacteria are used in disease prevention and biological control, such as pseudomonas aeruginosa, bacillus [4] [5]. Unfortunately, there is no research focused on functional bacteria interaction with endogenous microorganisms in oil reservoir.

Exogenous bacteria has a very good compatibility and can promote oil displacement recovery. Experimental outcome has showed that exogenous bacteria would compete with reservoir source microorganism [6]. As a result, the competition relationship between exogenous bacteria and reservoir endogenous bacteria is closely related to the microbial oil displacement effect. In the process of microbial oil displacement numerical simulation, it is vital to study the exogenous bacteria nutrition competition relations with endogenous bacteria.

For exogenous microbes flooding, current foreign typical mathematical models include Islam model, Zhang model and Chang model [7]. The Islam and Zhang models are mainly used in the theory and rock model study. In Chang model, the specific aspects about growth, death, adsorption, chemotaxis, nutrient utilization were described in detail for microorganisms and nutrients in reservoir, while the reaction kinetics model is relatively simple. Based on the above three models, Lei Guanglun and Gu Jianwei had established the product equilibrium equation in the mathematical model, and the physical parameters such as original viscosity, porosity, permeability enable the quantitative analysis for reservoir characteristics[8]. Zhu Weiyao had raised a multicomponent mathematical model, and it focused a significant part on limiting effect of nutrients by two microbial components without the interaction functional mechanism between them[9].

Current research mainly focused on simulated calculations about a single species. For injected function bacteria, reservoir compatibility could reflect the activity of microorganisms, which is the decisive factor for the successful improving recovery. Therefore, the particular interaction between injected features bacteria and endogenous bacteria were discussed in this paper, and the corresponding model equations were established in the study.

\section{Model Formulation Assumptions}

In microbial enhanced oil recovery (MEOR), biological behaviors like growth, death, chemotaxis, adsorption, nutrient consumption, product formation and stimulation principle were matched with the assumptions: The presence of three fluid phases (oil, gas and water) is considered; The volume of each phase would be combined, and the volume of oil and water are slightly compressible; Thermodynamics balance exist instantaneously, and extended law of Darcy is applied to the system of multi-phases; Bacteria and metabolites such as surfactant, polymer, and gases mainly contribute to oil displacement recovery; The reservoir is isothermal.

Seepage of oil and gas in reservoir conforms to laws of Darcy and mass conservation. Some researchers [10] had established the three dimensional-three phases seepage model governing equations for oil, water, gas phases.

\section{Microbial material balance equation}

In microbial oil displacement process, compatibility research of microbes and reservoir mainly centered the effects of reservoir parameters such as temperature, pressure, salinity on microbial growth by ignoring the influence of endogenous bacteria on injected bacteria [11]. Injected functional bacteria must have a good adaptability in oil reservoir. Microbial oil displacement recovery inevitably involves multiple microbial components. To reflect the interaction of different bacteria and to simplify the model, the microbial model equations mainly include two microbial components of injected bacteria and endogenous bacteria. Quantitative description was made for the microorganisms in convection diffusion, adsorption, migration, growth, death and other behaviors [12].

$$
\frac{\partial}{\partial t}\left(\frac{\varphi S_{w}}{B_{w}} C_{k 1}+\varphi C_{k 3}\right)=-\nabla\left(\frac{\bar{u}_{k t}}{B_{w}} C_{k 1}\right)+\nabla\left(\frac{\varphi S_{w}}{B_{w}} \overline{\bar{D}}_{k w} \nabla C_{k 1}\right)-\frac{q_{w}}{V_{b}} C_{k 1}+\frac{\varphi S_{w}}{B_{w}} R_{k}
$$




$$
\begin{aligned}
& k=1,2 \\
& u_{k t}=u_{w}+u_{g}+u_{k c} \quad k=1,2 \\
& u_{k}=u_{w} \quad k=3,4,5 \\
& \frac{\partial C_{k 3}}{\partial t}=R_{r}-R_{d}=K_{r}\left|\bar{u}_{w}\right| C_{k}\left(1-\sigma_{k}\right)-K_{d}\left(\sigma_{k} \rho_{k s c}\right)\left|\nabla \phi_{w}\right| C_{k} \quad k=1,2 \\
& C_{k 3}=\min \left(C_{k 3 \max }, \frac{a_{k} C_{k 1}}{1+b_{k} C_{k 1}}\right) \quad k=3,4,5 \\
& u_{g}=\frac{b+0.67}{b+(0.93 / \xi)} \frac{\left(\rho_{b}-\rho_{w}\right) g d_{b}^{2}}{18 \mu_{w}} \quad k=1,2 \\
& u_{k c}=k_{k c} \nabla \ln \left(C_{3}\right) \quad k
\end{aligned}
$$

In the type: $C_{k 1}$ is the concentration of each component in water phase, g/L; $C_{k 3}$ is the concentration of each component in adsorption phase, $\mathrm{g} / \mathrm{L} ; \overline{\bar{D}}_{k \mathrm{w}}$ is the convection diffusion coefficient, $\mathrm{m}^{2} / \mathrm{s} ;{ }^{u_{g}}$ and $u_{c}$ are sedimentation velocity of bacteria and chemotactic velocity for bacterial migration respectively, $\mathrm{m} / \mathrm{s} ; k_{c}$ is the chemical chemotactic coefficient, $\mathrm{m}^{2} / \mathrm{d} ; \quad b \approx 1 ; \xi$ denotes the empirical correction factor considering the influences of grain surfaces $(0 \leq \xi \leq 1) ; \rho_{\mathrm{b}}$ is biomass density, $\mathrm{g} / \mathrm{L} ; d_{\mathrm{b}}$ is the diameter of bacteria seen as a sphere, $\mathrm{m}$; $k_{\mathrm{r}}$ is adsorption factor, $\mathrm{m}^{-1}$; $k_{d}$ is desorption coefficient, $\mathrm{m} /$ (MPa.h) $; \sigma$ is pore volume fraction occupied by microbial adsorption; $\mathrm{a}_{k}$ and $\mathrm{b}_{k}$ are adsorption constants for component $\mathrm{k} ; B_{w}$ is the formation volume factor; $\phi_{\mathrm{w}}$ is water potential energy, $\mathrm{J}$; b is the subscript indicating the microbial component.

\section{Growth Speed Equations of Reaction Dynamics}

When functional bacteria and nutrients are injected into the formation underground together, endogenous bacteria and functional bacteria would compete for nutrition at the same time. There is also a product of symbiotic relationship for the two kinds of bacteria, and symbiosis mechanism is difficult to present in the performance among the concentrations changes from by-products or related products. Based on Monod model, maximum specific growth rate was modified to simplify the model and to express competitive relationship between the two microbial components, and the substrate inhibition constant was revised to reflect the symbiotic relationship [13].

$$
\begin{aligned}
& \mu_{g 1}=\frac{\alpha_{12} \mu_{m 1} C_{3}}{\beta_{12} K_{s 1}+C_{3}} \\
& \mu_{g 2}=\frac{\alpha_{21} \mu_{m 2} C_{3}}{\beta_{21} K_{s 2}+C_{3}} \\
& \mu_{d 1}, \mu_{d 2}=\text { const } \\
& r_{i}=\left(\mu_{g i}-\mu_{d i}\right) \quad i=1,2
\end{aligned}
$$

Equations for Nutrient Consumption Rate

$$
r_{3}=\sum_{j=1}^{2} \frac{1}{Y_{j}} \mu_{j}+\sum_{j=1}^{2} m_{s j}
$$

Equations for Product Generation Rate 


$$
r_{i}=\sum_{j=1}^{2} \lambda_{i j} C_{j}+\sum_{j=1}^{2} \eta_{i j} \frac{d C_{j}}{d t} \quad i=4,5
$$

Therefore, reaction rate equation is shown as following:

$$
R_{i}=r_{i}\left(C_{k 1}+\sigma \rho_{b}\right) \quad i=1,2, \cdots 5
$$

In the type: $C_{1}$ and $C_{2}$ are thallus concentration for component 1 and component 2 respectively, $\mathrm{g} / \mathrm{L} ; \mu_{m 1}$ and $\mu_{m 2}$ are the maximum specific growth rate for component 1 and component 2 respectively, $1 / \mathrm{s} ; \quad k_{s 1}$ and $k_{\mathrm{s} 2}$ are half-saturation coefficient, $\mathrm{g} / \mathrm{L} ; \eta_{41}, \eta_{42}, \eta_{51}, \eta_{52}$ are metabolites generation rate for bacteria to sustain life; $\lambda_{41}, \lambda_{42}, \lambda_{51}, \lambda_{52}$ are metabolic product yield rate for different components; $Y_{1}$ and $Y_{2}$ are bacteria yield rate; $m_{\mathrm{s} 1}, m_{\mathrm{s} 2}$ are maintain factor, $1 / \mathrm{s} ; \alpha$ and $\beta$ are interaction factors of two microbial components; if the two bacteria are in a state of symbiotic relationship, then $\alpha>1, \beta<1$; if the two kinds of bacteria are in a state of repressive relationship, then $\alpha<1, \quad \beta>1$; if the two bacteria do not interfere each other in growth just like a single bacteria, then $\alpha=\beta=1$; letters of $\mathrm{g}$ and $\mathrm{d}$ are subscripts meaning growth and death respectively.

Mechanisms for MEOR mainly comprise the transformation of rock, fluid and seepage rule from microorganism. According to these mechanisms, roles of bacteria itself and its metabolites were considered comprehensively as shown in table 1.

Table 1 Action mechanisms for microorganism and major metabolites in the model

\begin{tabular}{cl}
\hline Types for products & Treatment measures in the model \\
\hline \multirow{2}{*}{ organism } & as a single component \\
& microorganism concentration $\sim$ crude oil viscosity (degradation) \\
& microorganism concentration $\sim$ absolute permeability （ resistance \\
& coefficient $)$ \\
& as a single component \\
& surfactant $\sim$ interfacial tension \\
& interfacial tension $\sim$ capillary force \\
& capillary force $\sim$ residual oil saturation \\
biosurfactant & residual oil saturation $\sim$ relative permeability \\
\hline & as a single component \\
& biopolymer $\sim$ water phase viscosity \\
& biopolymer $\sim$ absolute permeability (resistance coefficient $)$ \\
\hline biopolymer & as a single component \\
& gas-oil ratio $\sim$ crude oil viscosity \\
\hline
\end{tabular}

\section{Absolute Permeability Transformation}

Microbial retention on pore surface could decrease the porosity and permeability of formation rock. Decline for permeability is mainly caused by tiny particles and metabolic product, therefore, permeability decline mainly comes from two parts, one factor refers to the microbial cells function on permeability. Decrease of permeability is proportional to the third power of reduction from porosity. When the pore throat was jammed in porous media, the change of porosity may not be significant, but the permeability will be greatly reduced, so a flow efficiency coefficient $\mathrm{F}$ was amended, and $\mathrm{F}$ is mainly determined by the pore diameter distribution of bimodal function. Another impact factor is the permeability affected by microbial metabolites (acid, $\mathrm{CO}_{2}$, polymer).

$$
\varphi^{\prime}=\varphi-\sum_{k=1}^{2} \sigma_{k} \quad k=1,2
$$




$$
\begin{aligned}
& \frac{K^{\prime}}{K}=F\left(\frac{\varphi}{\varphi^{\prime}}\right)^{3} \\
& R_{k}=1+\frac{\left(R_{k \max }-1\right)}{1+b_{r k} C_{k}} \quad k=1,2
\end{aligned}
$$

In the type: $\varphi$ and $\varphi^{\prime}$ are the porosity before and after microbial action respectively; $K, K^{\prime}$ are the absolute permeability before and after microbial action respectively, $\mu m^{2} ; F$ is the factor for flow efficiency; $R_{k}$ is the coefficient of permeability decline; $R_{k \text {,max }}$ is the coefficient of maximum permeability decline; $b_{r k}$ is the undetermined constant.

\section{Viscosity Transformation}

In the process for MEOR, microorganisms and their metabolic products will have an effect on oil viscosity, like crude oil viscosity reduction caused by microorganisms metabolic degradation, surfactant emulsion and gases $\left(\mathrm{CO}_{2}, \mathrm{CH}_{4}\right)$ dissolution. Water viscosity was mainly acted by injected nutrition system and metabolism of polymer material. The rules can be determined by fermentation experiment of microorganism and crude oil. Viscosity transformation equation was shown as following.

$$
\begin{aligned}
& \mu_{o}^{\prime}=\mu_{o}\left(C_{1}, C_{2}, C_{3}, C_{4}, C_{6}\right) \\
& \mu_{w}^{\prime}=\mu_{w}\left(C_{3}, C_{5}\right)
\end{aligned}
$$

In the type: $\mu_{o}$ and $\mu_{o}^{\prime}$ are oil viscosity before and after microbial action respectively, $\mathrm{mPa} \cdot \mathrm{s}$; $\mu_{w}$ and $\mu_{w}^{\prime}$ are water viscosity before and after microbial action respectively, $\mathrm{mPa} \cdot \mathrm{s}$.

\section{Relative Permeability Transformation}

In addition to the transformation of wettability, interfacial tension and other physical parameters, a stronger chemical surfactant emulsification can occur under the condition of low speed stirring in the process of microbial cultivation. Relative permeability changed and residual oil saturation reduced eventually. According to the synergistic effect for residual oil recovery, residual oil saturation equation was modified by microbial factor to reflect the synergy of interfacial tension, wetting and emulsifying comprehensively.

$$
\begin{aligned}
& N_{c}=\frac{\left|\sum_{j=1}^{2} \mu_{j} u_{j}\right|}{\sigma_{w o}} \quad j=w, o \\
& \ln S_{o r}=d_{1}\left(\ln k_{b} N_{c}\right)^{2}+d_{2} \\
& K_{r w}=K_{r w}^{0}\left(\frac{S_{w}-S_{w i}}{1-S_{o r}-S_{w i}}\right)^{n_{1}} \\
& K_{r o}=K_{r o}^{0}\left(\frac{1-S_{w}-S_{o r}}{1-S_{o r}-S_{w i}}\right)^{n_{2}}
\end{aligned}
$$

In the type: $\quad k_{b}$ is the microbial factor; $d_{1}$ and $d_{2}$ are constants; $N_{c}$ is capillary number; $\sigma_{\text {ow }}$ is oil-water interfacial tension, $\mathrm{mN} / \mathrm{m} ; S_{\text {or }}$ is residual oil saturation, dimensionless; $S_{w}$ is water saturation, dimensionless; $S_{w i}$ is bound water saturation, dimensionless; $K_{r w}$ and $K_{r o}$ are relative permeability for water and oil phases respectively, dimensionless; $K_{r w}^{0}$ and $K_{r o}^{0}$ are endpoint relative permeability for water and oil phases respectively, dimensionless; $n_{1}$ and $n_{2}$ are power exponent in known curves. 


\section{Conclusion}

A new MEOR mathematical model was established in this paper, and it is suitable for functional bacteria in three-dimensional three-phase multicomponent. Two microbial components interacting factors was introduced to reflect the interaction of injected functional microbes with endogenous microorganisms, which helps make up reaction kinetics equation in MEOR mathematical model.

By taking production mechanism of MEOR into account, the mathematical model has expounded the transformation for absolute permeability, viscosity and relative permeability successfully to guarantee its application.

\section{Acknowledgement}

The authors would like to thank the research institute of Civil and Environmental Engineering School, University of Science and Technology Beijing for the support. This paper is sponsored by the national high technology research and development program 863 Project (No.2009AA063504).

\section{References}

[1] Jing Guicheng, Liu Fuhai, Guo Shangping. Mechanism of enhanced oil recovery by loca 1 microbial enrichment [J]. Acta Petrolei Sinica, 2004.9, 25(5):70-74.

[2]Bao Mutai,Wang Weidong,Wang Xiulin. Microbial Enhanced Oil Recovery by Activation of Stratal Microflora: a Review [J]. Oilfield Chemistry, 2012, 19(4):382-386.

[3]Ma Zijian,GaoYujun,SuYe.Application of PCR-DGGE techonology in Microbial Enhanced Oil Recovery [J]. Inner Mongolia petrochemical industry, 2001 (2):118-119.

[4]Chen Tianyou, Dong Siguo, Tian Wanhong. Effect of Bacillus subtilis Strain BS23 Inhibi ting the Growth of Six Enteric Bacteria [J]. Journal of Microbiology. 2004 (5):74-76.

[5]Sun Jiquan,TangYueqin,LiuWeiqiang.Characteristics of degradation of phenolic compounds by two Pseudomonas strains [C]. China Environmental Science. 2010 (12):1633-1638.

[6]Zhang Zhongzhi, Li Qingzhong, Wang Hongjun. Advances in Researches on Field Trials for Microbial Enhanced Oil Recovery [J]. Oilfield Chemistry.2001 (4):383-388.

[7]Islam M P. Mathematical modeling of microbial enhanced oil recovery. SPE 204801990

[8]Lei Guanglun, Chen Yueming, Gao Lianyi. Mathematical models of Microbial Flooding [J]. Journal of the University of Petroleum,China. 2001 (2): 46-49.

[9]Zhu Weiyao,Yang Zhengming,Chi Li.A microbial transport compositional model simulator [J]. Petroleum Exploration and Development. 2000 (3): 44-46.

[10]Yuan Shiyi, Wang Jialu. Petroleum Reservoir Numerical Simulation. Beijing: Petroleum industry press, 2004:11-17.

[11]Yang Chengzhi, Lou Chuhong. Geological Basis and Selection Criteria for Microbial oil Recovery [J]. Petroleum Exploration and Development. 1997.4

[12]Xiu Jianlong, Yu Li, Guo Ying. A mathematical coupling model of seepage field and microbial field in the indigenous microbe enhancing oil recovery [J]. Acta Petrolei Sinica. 2010(6):989-992.

[13]Zhang Xuehong, Tang Yonglian, Huang Ruishan. Growth Kinetics of Mixed Cultures of Bifidobacterium Adolescentis and Streptococcus Lactis [J]. Journal of Shanghai Jiaotong Uni versity. 1997 (4):82-85. 